\title{
Editorial of the special issue on Advances in Low-Level Image representations for processing and analysis
}

\author{
Abderrahim Elmoataz $^{1} \cdot$ Fathallah Nouboud $^{2} \cdot$ Olivier Lézoray $^{1} \cdot$ Driss Mammass $^{3}$
}

Received: 25 July 2015 / Accepted: 28 July 2015 / Published online: 2 August 2015

(C) Springer-Verlag London 2015

\section{Background and motivation}

The representation of visual information present at specific locations in images is a fundamental task in image processing analysis. Having a suitable representation for visual features enables reasoning with the end goal of helping object categorization, image region classification, image segmentation and restoration among others. Several low-level image representations have proven their benefits, one can quote wavelets, Bag Of Words, sparse representations, Local Binary Patterns, etc. The importance of such image representation is now prevalent in image processing and analysis. This issue aims at presenting recent developments in this area from both theoretical and application perspectives to capture the widest possible range. We hope that the reader will enjoy the interesting research works selected for this special issue.

Olivier Lézoray

olivier.lezoray@unicaen.fr

Abderrahim Elmoataz

abderrahim.elmoataz-billah@unicaen.fr

Fathallah Nouboud

Fathallah.Nouboud@uqtr.ca

Driss Mammass

mammass@uiz.ac.ma

1 GREYC UMR 6072 CNRS - Image Team, Université de Caen Normandie, Caen, France

2 Département des Mathématiques et Informatique, Université du Québec à Trois-Rivières, Quebec, Canada

3 Université Ibn Zohr, Agadir, Morocco

\section{Quick facts about the special issue}

The guest editors suggested putting together this special issue to the editor-in-chief in August 2013. In September 2013, the guest editors and the editor-in-chief established the outline and schedule of the special issue. This special issue presents extended version of the best papers of the 2014 International Conference on Image and Signal Processing on the specific topic of "Advances in Low-Level Image representations for processing and analysis." The ICISP 2014 conference took place in Cherbourg-Octeville (Normandy, France) from June 30 to July 2, 2014. The general and program committee chairs of ICISP 2014 have selected the 10 best papers on the selected topic. In July 2014, authors of selected papers were invited to submit an extended version of their paper to this special issue. Each of the papers was reviewed by two or three experts in the field. After two rounds of rigorous reviews between July 2014 and July 2015, nine papers were finally accepted for inclusion in the special issue.

\section{Scanning the special issue}

Papers included in this special issue focus on various topics, and the special issue is organized around these.

\subsection{Color}

In their paper entitled "Contribution of color in saliency model for videos," Hamel et al. investigate the contribution of color information in a bottom-up visual saliency model. Such bottom-up saliency models have been developed so far to predict the location of gaze according to low-level features of a visual scene. However, the contribution of color in guiding eye movements is still unclear. Using an eye-tracker, 
they show that color information improves the performance of their saliency model in predicting eye positions.

In their paper entitled "Colour and Multiscale Texture Features from Vectorial Mathematical Morphology," Ledoux et al. explore a way to compute texture features for color images within a complete vectorial process. Using a dedicated approach for color ordering, they produce a complete framework for color mathematical morphology adapted to human visual system characteristics. Morphological multiscale texture features are defined and their performances exhibited on reference texture datasets.

\subsection{Filtering}

In their paper entitled "Nonlocal PDEs Morphology: A Generalized Shock Operator on Graph," Sadi et al. present an extension of shock filters on weighted graphs using the formalism of partial difference equations. This adaptation leads to new morphological operators that alternate between nonlocal dilation and nonlocal erosion filter type on graphs. The approach is illustrated on various types of data represented by graphs.

In their paper entitled "Removal of Poisson noise in hyperspectral images using a spatial-spectral adaptive total variation approach," Deger et al. propose a variational-based denoising approach for multi- and hyperspectral images. Their approach accounts both the vectorial structure of a spectral image cube and Poisson noise. A specific regularization term that is spectrally and spatially adaptive is introduced with a split Bregman optimization.

\subsection{Recognition}

In their paper entitled "Identification of precancerous lesions by multispectral gastroendoscopy," Martinez Herrera et al. propose a minimally invasive technique based on multispectral imaging and a methodology to identify malignancies in the stomach. They have developed a multispectral gastroendoscopic system compatible with current gastroendoscopes. The spectra are extracted from the acquired multispectral images in order to compute statistical features classified into benign and malign.

In their paper entitled "Gait Recognition Based on Modified Phase Only Correlation," Rida et al. consider the problem of identifying people purely through the analysis of the way they walk. They propose a supervised relevant feature extraction method for human recognition that mitigates the problems due to clothing variations.

In her paper entitled, "A Comparative Study of Irregular Pyramid Matching in Bag-of-Bags of Words Model for Image Retrieval," Yi Ren compares three standard approaches to build irregular pyramid partitions built using graph partitioning methods. She extends the bag-of-words model to a bag-of-bags of words model by embedding color homogeneity and limited spatial information through irregular partitions of an image.

\subsection{Detection and tracking}

In their paper entitled "Energy-Transfer Features Combined with DCT for Object Detection," Fusek et al. propose an improvement of the sliding window detector that is based on energy-transfer features. The basic idea of these features is that the appearance (shape) of objects can be described using energy distribution function. Using this new approach, object can be efficiently encoded with a short vector and enables good detection results regarding state-of-the-art methods.

In their paper entitled "Sparsity in Optical Flow and Trajectories," Gibson et al. apply sparse constraints to improve optical flow and trajectories. They apply sparsity in two ways. First, with two-frame optical flow, they enforce a sparse representation of flow patches using a learned over-complete dictionary. Second, they apply a low-rank constraint to trajectories via robust coupling.

Acknowledgments The guest editors thank all those who have helped to make this special issue possible, especially the authors and the reviewers of the articles. They thank the editorial staff for her help and support in managing the issue and, finally, gratefully acknowledge the EditorIn-Chief for giving them the opportunity to edit this special issue. 